\title{
In vitro and in vivo Biological Responses to Graphene and Graphene Oxide: A Murine Calvarial Animal Study
} This article was published in the following Dove Press journal:
International Journal of Nanomedicine

Background: Graphene and its derivatives have recently gained popularity in the biomedical field. Previous studies have confirmed that both the mechanical strength and wear resistance of graphene-containing polyethylene have been greatly improved. Therefore, it is being considered as an alternative for artificial joint replacement liners. Based on the literature, the wear debris generated from the traditional polymers used for orthopedic liners could lead to particle-induced osteolysis and, consequently, failure of joint replacement. However, the biological response of this novel graphene-based polymer is still unclear. Therefore, the current study aimed to investigate the in vitro and in vivo biological effects of graphene and graphene oxide (GO) particles on bone.

Materials and Methods: The biological responses of graphene and GO particles were tested via in vitro and murine calvarial in vivo models. In the in vitro model, murine macrophage cells were mixed with particles and hydrogel and printed into two differently designed scaffolds; the induced proinflammatory cytokines were then tested. In the murine in vivo model, the particle size distribution was measured via SEM, and these particles were then administrated in the calvarial area, referring to our established model. A micro-CT and histological analysis were performed to examine the biological effects of the particles on bone health. The data were analyzed via the one-way analysis of variance to determine the differences between the groups.

Results: Both graphene and GO induced significantly higher TNF- $\alpha$ and IL-6 secretion compared with the control in the three-dimensional in vitro model. In the murine calvarial in vivo test, the graphene and GO particles increased the bone mass compared with the sham groups in the micro-CT analysis. Bone formation was also observed in the histological analysis. Conclusion: In these in vivo and in vitro studies, the graphene and GO wear debris did not seem to induce harmful biological response effect to bone. Bone formation around the skull was observed in the calvarial model instead. Graphene-containing biomaterials could be a suitable new material for application in orthopedic prostheses due to their benefit of eliminating the risk of particle-induce osteolysis.

Keywords: biological response, graphene, graphene oxide, osteolysis, osteogenesis

\section{Introduction}

Joint replacement for the treatment of severe joint deformation caused by degeneration has become a common orthopedic surgery. The success rate after replacement and the postoperative functional performance of patients are satisfactory. ${ }^{1,2}$ However, wear deformation of the joint components or wear of the material can induce osteolysis and aseptic loosening, resulting in failure after a long period of
Correspondence: Chang-Hung Huang Department of Medical Research, MacKay Memorial Hospital, No. 45, Minsheng

Road, Tamshui District, New Taipei City,

Taiwan

Email changhung08I2@gmail.com 
use. This problem is still an intractable clinical condition in the orthopedics field. ${ }^{3,4}$ In particular, the issue regarding the wear of ultra-high molecular weight polyethylene (UHMWPE) still needs to be overcome.

To improve the wear resistance of these materials, scientists have developed highly crosslinked polyethylene (HXLPE) through the alteration of process technology. 5,6 Although the wear resistance has improved, the crosslinking process leads to a decline in mechanical properties, which is a major disadvantage. ${ }^{7,8}$ For the third generation of orthopedic liners, to prevent oxidation and decline of mechanical properties during the crosslinking process, antioxidants such as vitamin $\mathrm{E}^{9}$ and COVERNOX ${ }^{10}$ have been added to HXLPE. Since antioxidant-incorporated polyethylene was clinically introduced just a few years ago, there have only been short-term clinical follow-up studies thus far. ${ }^{11,12}$ Researchers are still looking for more suitable materials to use for orthopedic liners.

Graphene is a novel material that has been gaining attention in the biomedical research industry in recent years. ${ }^{13}$ Graphene and its derivatives are already used in biomedicine for antibacterial, gene therapy, immunodiagnostic wafers, image detection, and drug release carriers. ${ }^{14,15}$ Graphene is a two-dimensional (2D) single-atom-thick sheet of $s p^{2}$ hybridized hexagonally arranged carbon atoms within a carbon material structure. ${ }^{16}$ It has been demonstrated to possess various properties including electrical conductivity, ${ }^{17}$ high elasticity, ${ }^{18}$ high strength, ${ }^{17}$ and flexibility. ${ }^{16}$ Meanwhile, graphene oxide (GO), a graphene derivative, has different electronic structures from graphene. ${ }^{19}$ It has a mixed structure carrying various oxygencontaining functional groups such as the epoxy $(>0)$, hydroxyl $(\mathrm{eOH})$, carbonyl $(\mathrm{C}=\mathrm{O})$, and carboxyl (-COOH) groups. ${ }^{20,21}$ These functional groups allow different biomolecules, including proteins, DNA, and RNA, to efficiently attach to $\mathrm{GO}^{15}$ while also making it hydrophilic. ${ }^{22}$

Due to their osteogenic and high mechanical characteristics, different forms of graphene and GO have been used in research for various purposes, including coating on metal disks, ${ }^{23,24}$ blending with polymers, ${ }^{25}$ and mixing with polymer pellets to form three-dimensional (3D) scaffolds with 3D printers. ${ }^{26}$ There is also a novel form of graphene called graphene foam; 3D graphene foam is made via chemical vapor deposition processing. It has been characterized as a new class of highly conductive graphene-based materials with excellent mechanical strength, flexibility, and elasticity. Furthermore, its high surface area promotes cell adhesion and growth. ${ }^{27,28}$
However, Pang et $\mathrm{al}^{29}$ tested the biosafety of graphene and GO in vitro and in vivo. In their study, graphene and GO nanoparticles were cocultured with rat PBMNCs for $24 \mathrm{hrs}$, and it was found that $500 \mu \mathrm{g} / \mathrm{mL}$ of GO reduced the cell viability to only about $40 \%$, while the survival rate of cells cocultured with higher concentrations of graphene $(1,000 \mu \mathrm{g} / \mathrm{mL})$ was only reduced to about $80 \%$. To simulate the effect of orthopedic implants on the body, graphene and GO nanoparticles were injected into mouse skeletal muscle for 7 days. In the histology analysis, the pure graphene particles had no obvious effects on the skeletal muscle, while GO caused severe muscle atrophy and necrosis. It has also been found that graphene can induce cytotoxicity by lowering the mitochondrial membrane potential and increasing intracellular reactive oxygen species. ${ }^{30}$ However, it has a strong adsorption capacity to adsorb serum protein and form protein inclusions, exhibiting a higher cytotoxicity compared with that of graphene. $^{31}$

With the latest manufacturing technology, after evenly mixing GO powder with UHMWPE powder, GO-PE bars can be cast at a high temperature and high pressure. ${ }^{25}$ The results of various mechanical tests have shown that the mechanical strength increases as the content of GO increases. Compared with the tensile strength of conventional polyethylene, the Young's modulus, lodging strength, and ultimate strength are increased from $601.32 \pm 27.81 \mathrm{MPa}, 23.45 \pm$ $1.21 \mathrm{MPa}$, and $32.77 \pm 3.53$, respectively, to $664.38 \pm 28.32$ $\mathrm{MPa}, 24.57 \pm 1.19 \mathrm{MPa}$, and $36.91 \pm 3.98 \mathrm{MPa}$, respectively.

Due to its high mechanical properties, GO could become a candidate for the development of new materials for artificial joint replacement liners. However, the biological effects on bone of graphene or GO debris are still unclear. In the current study, we use our previously established in vivo mice calvarial osteolysis model to test the effects of new biomaterial particles on the biological response to the bone interface. ${ }^{32}$ This study aims to investigate the in vitro and in vivo effects of graphene and GO particles via our previously developed 3D-printed in vitro and mice calvarial in vivo osteolysis models. ${ }^{33,34}$

\section{Materials and Methods}

\section{Particle Preparation}

Graphene (AL-900394) and GO nanoparticles (AL795534) were purchased from Sigma-Aldrich (St. Louis, MO, U.S.A.). Polystyrene (PS) particles (Polystyrene Latex Spheres, 610-38) were purchased from Ted Pella, Inc. (CA, 
U.S.A.). Although polyethylene is the material used for joint replacement inserts, PS particles were used for osteolysis in the in vivo model. ${ }^{35}$ The mean particle size was measured via scanning electron microscope (SEM; HITACHI S-3500N, JAPAN) images, whereby 300 particles were randomly selected for measurement of their size and aspect ratio. ${ }^{36}$ Their perimeter and long axis (particle size) were measured by the SEM built-in software to determine the aspect ratio.

To avoid possible endotoxin effects, the particles were washed with $75 \%$ ethanol, centrifuged for $45 \mathrm{~min}$ three times, and then transferred to $95 \%$ ethanol before being centrifuged another three times for $45 \mathrm{~min}$, according to our previous experience. ${ }^{32,34}$ The endotoxin level of all particles was tested via a limulus amebocyte lysate assay kit (ToxinSensor ${ }^{\mathrm{TM}}$ Gel Clot Endotoxin Assay Kit, GenScript, NJ, USA) to ensure an endotoxin level below $0.1 \mathrm{EU} / \mathrm{mL}$ for the in vitro model and below $0.25 \mathrm{EU} / \mathrm{mL}$ for the animal experiment (according to the FDA's published guidelines for LAL testing). ${ }^{37}$ The particles were then suspended in hyaluronic acid (HA) for animal surgery.

\section{Cell Culture}

The RAW264.7 murine monocytic/macrophagic cell line (ATCC, TIB-71, Manassas, VA, USA) was used in the current study. It has been used in osteolysis in vitro models in many other studies. ${ }^{38,39}$ The cells were cultured in Dulbecco's Modified Eagle Medium (DMEM) (Gibco, Thermo Fisher Scientific, Waltham, MA, USA) supplemented with $1 \%$ penicillin/streptomycin (Gibco) and $10 \%$ fetal bovine serum (Gibco) at $37^{\circ} \mathrm{C}$ in a humidified incubator with $5 \% \mathrm{CO}_{2}$. The cells were then harvested using a $0.05 \%$ trypsin ethylenediaminetetraacetic acid (EDTA) solution (Gibco) for $10 \mathrm{~min}$ and washed with Dulbecco's phosphate-buffered saline (PBS) (Gibco); viable cells were counted using trypan blue dye exclusion before seeding.

\section{Cell Laden Hydrogel Preparation}

Methylcellulose (MC) hydrogel was purchased from Hsintai Integration Biomed Ltd. (Hsintai Integration Biomed Ltd., Hsinchu, Taiwan). It was used in cell sheet harvest systems and bioengineered cardiac patches constructed from multilayered mesenchymal stem cells. ${ }^{40,41}$ The MC hydrogel has been applied for 3D bioprinting cell system including of cell sheet and cell spheroid production. ${ }^{42}$ In the current study, the MC hydrogel was prepared by dissolving MC powder
(Sigma-Aldrich, M7027, 15Cps) into sterilized PBS (12\% $\mathrm{w} / \mathrm{v}$ ) and kept cool before use.

Distinct cells and GO were mixed with MC hydrogel before being printed. The concentration of particles in the MC hydrogel was $2 \mathrm{mg} / \mathrm{mL}$. The murine macrophage cell line RAW264.7 was seeded at $1.5 * 10^{3}$ cell/well (structure) and the graphene or GO particles at $2 \mu \mathrm{g} /$ well (structure). All control groups contained cells and $\mathrm{MC}$ hydrogel but no particles.

\section{Bioprinting of 3D Structures}

Multiple layers of a 3D structure culture system were printed by a modified 3D bioprinter. Two different 3D structures, a grid and a honeycomb, were tested in this model (Figure 1). The grid structure was a square of $6 \mathrm{~mm}$ on all sides. The honeycomb structure was combined with seven hexagons with a length of $2 \mathrm{~mm}$. Both structures were printed with five 2-mm-thick layers. The cells and particles were mixed together with MC hydrogel before being printed. A 2D control was performed in a 24-well culture plate. The structures were cultured in a 12-well plate with DMEM after being printed. All groups were cultured for $72 \mathrm{hrs}$ at $37^{\circ} \mathrm{C}$ in a $5 \% \mathrm{CO}_{2}$ incubator.

\section{Assessment of Proinflammatory Cytokines}

Supernatants from the cell/particles culture were collected after $72 \mathrm{hrs}$ of culture. The concentrations of specific cytokines (murine TNF- $\alpha$ and IL-6) were assayed using commercially available enzyme-linked immunosorbent assay kits (ELISA, PeproTech, Rocky Hill, NJ, USA for TNF- $\alpha$ and Biolegend, San Diego, CA, USA for IL-6).

\section{Animals and Surgical Treatment}

The protocol for this experiment was approved by the Institutional Animal Care and Use Committee of MacKay Memorial Hospital, where the study was performed. All animal procedures were performed by the Guideline for the care and use of laboratory animals by Council of Agriculture, Executive Yuan, Taipei, Taiwan. A total of 24 eight-week-old C57BL/6J female mice were purchased from the National Laboratory Animal Center, Taipei, Taiwan, an AAALAC International certified biotechnology company. The animals were kept in a room at $24^{\circ} \mathrm{C}$ and $50 \%$ humidity, with a $12 \mathrm{hr}$ light/dark cycle (light from 7:00 a.m. to 7:00 p.m.). These in vivo particleinduced osteolysis calvarial experiments were established 
A

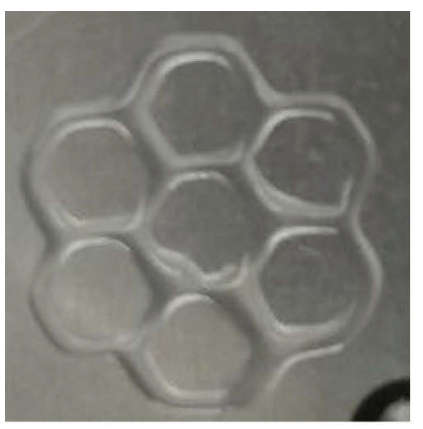

D

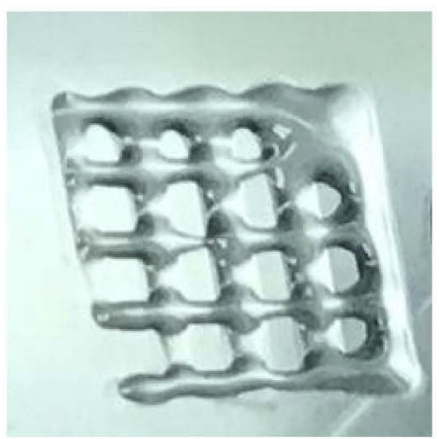

B

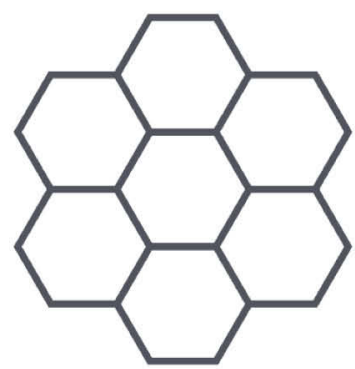

E

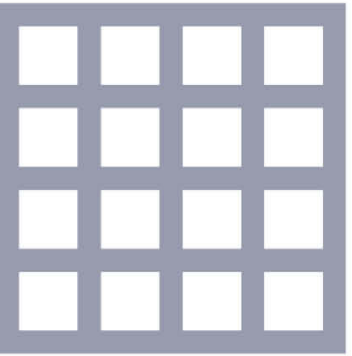

C

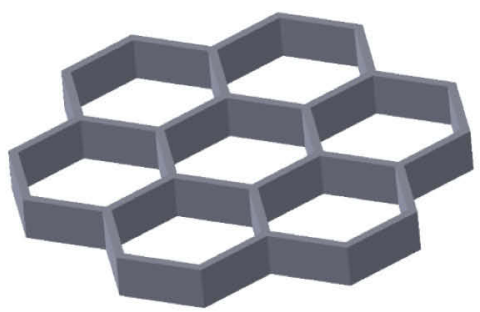

$\mathbf{F}$

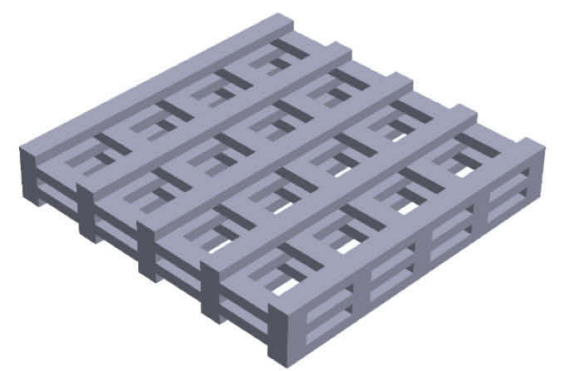

Figure I Images of in vitro 3D structures. (A) 3D-printed honeycomb structure; (B) simulated top view of honeycomb structure; (C) simulated side view of honeycomb structure; (D) 3D-printed grid structure; (E) simulated top view of grid structure; $(\mathbf{F})$ simulated side view of grid structure.

in our previous studies and used to determine the biological effect of different novel biomaterial particles on calvarial bone. ${ }^{32,34}$ The animals were randomly separated into four groups: (1) sham group $(n=6)$ (underwent sham surgery only); (2) PS particle group $(\mathrm{n}=6)$; (3) graphene group $(\mathrm{G}, \mathrm{n}=6)$; and (4) GO group $(\mathrm{n}=6)$. Moreover, HA was used as a carrier to keep the particle solution within the incision after surgery. This carrier was a consistent way to establish the murine calvarial osteolysis model in our study. The PS, graphene, and GO particles were resuspended via ultrasonication in HA with a concentration of $1 \mathrm{mg}$ particles $/ 100 \mu \mathrm{L} .^{32,34}$ The mice were anesthetized with $100 \mathrm{mg} / \mathrm{kg}$ Zoletil 50 and $10 \mathrm{mg} / \mathrm{kg}$ Rompun by intraperitoneal injection. A $0.5 \times 0.5-\mathrm{cm}$ area of the middle sagittal incision of the calvaria was exposed. After removing the periosteum intact, the particle suspensions were spread over the area with a $25-G$ needle, and the incision was closed with sutures. ${ }^{32}$ All groups were analyzed by micro-CT before surgery (zero weeks) and 2 ,
4, and 6-week postsurgery. All animals were sacrificed in the sixth week of the treatment.

\section{Micro-CT Imaging Analysis}

The live animals were analyzed via micro-CT at 0,2 , and 4 weeks postsurgery by Micro-CT (Skyscan 1176 Bruker Micro-CT, Kontich, Belgium). After 6-week postsurgery, the calvarias with skin were fixed in $10 \%$ buffered formalin for $24 \mathrm{hrs}$ and then transferred to $70 \%$ ethanol for another $24 \mathrm{hrs}$ before scan. The micro-CT was set in a $2048 \times 2048$ pixel matrix. The 3D images were reconstructed with a voxel size of $9 \mu \mathrm{m}$. For quantitative analysis of particle-induced osteolysis, a spherical volume of interest (VOI) of $5 \mathrm{~mm}$ in diameter was defined with the bregma as the center to measure the differences in the bone mineral density (BMD, $\mathrm{mg} / \mathrm{cc}$ ), ratio of bone volume to tissue volume (BV/TV, \%), and trabecular thickness (Tb.Th) between each group. 


\section{Histological Analysis}

The calvarias were decalcificated in 10\% EDTA for 2 weeks and paraffin embedded. Five $\mu \mathrm{m}$ thick sections of each calvaria were taken from the sagittal plane centered over the area of particle-induced osteolysis. The sections were collected for hematoxylin and eosin staining as a general overview to observe the pro-inflammation morphology of the connective tissue. Masson trichrome staining was used to identify fibrous tissue and new bone formation. Tartrateresistant acid phosphatase (TRAP) staining was performed using a commercial TRAP kit (\#386A, Sigma-Aldrich). The number of osteoclasts was determined via counting the number of TRAP-positive multinucleated cells by two co-authors that were blinded to the data.

\section{Statistical Analysis}

The data were first analyzed with a normality test and showed a normal distribution. Then, they were analyzed by the oneway analysis of variance to determine the differences between the groups. Multiple comparisons were adjusted with a Bonferroni posthoc test. The results were reported as mean \pm standard deviation (SD). A $p$-value less than 0.05 was considered significantly different.

\section{Results}

\section{Particle Morphology}

The graphene nanoplatelets were $1.18 \pm 0.62 \mu \mathrm{m}$ (mean size) and $1.44 \pm 0.35$ (aspect ratio). The PS particles were $1.03 \pm 0.04 \mu \mathrm{m}$ (mean size) and $0.99 \pm 0.03$ (aspect ratio) (Figure 2).

\section{Cell Response to Graphene and GO in 3D Osteolysis in vitro Model}

Both graphene and GO induced significantly higher TNF- $\alpha$ and IL-6 secretion compared with the control in the $3 \mathrm{D}$ in vitro model (Figure 3). The graphene group cultured in the 3D model was approximately $0.6-2.8$ folds and 1.4-2.6 folds elevated of TNF- $\alpha$ and IL- 6 compared with those of 3D control structure, respectively. The GO group cultured in the 3D model was approximately 1.7-3.9 folds and 1.3-5 folds elevated of TNF- $\alpha$ and IL- 6 compared with those of $3 \mathrm{D}$ control structure, respectively.

\section{Micro-CT Imaging Analysis}

An in vivo micro-CT analysis was performed on the mice to track whether the graphene family particles induced osteolysis during the preoperative ( 0 weeks) and two, four, and six weeks postoperative periods. The reconstruction of the calvaria images by $3 \mathrm{D}$ view showed that the G group had no obvious osteolysis reaction in weeks four and six (Figure 4A). The GO group had pits on the calvaria at week 2 , but the pits decreased at week 4 and new bone formation was observed. Six weeks after the surgery, the bone formation in the GO group was more obvious than that in the other groups.

The comparison of the BMD results showed that the graphene family significantly increased the BMD value compared with the sham group at 2 and 4 weeks after the surgery (week two: $0.033 \pm 0.012$, week four: $0.035 \pm 0.011$ ). The G group increased the BMD by $103 \%$ and $138 \%$ in week two $(0.067 \pm 0.023)$ and week $4(0.081 \pm 0.022)$ (Figure $4 \mathrm{~B})$, respectively, while the GO group only increased it by $45 \%$ and $51 \%$ in week two $(0.048 \pm 0.012)$ and week $4(0.053 \pm$ $0.017)$, respectively. The $\Delta \mathrm{BV} / \mathrm{TV}$ results were similar to those of $\triangle \mathrm{BMD}$; the $\mathrm{BV}$ was significantly increased in the G group (week two: $4.14 \pm 0.94$, week four: $5.26 \pm 1.24$ ) and GO group (week two: $4.14 \pm 0.94$, week 4: $5.26 \pm 1.24$ ) compared with the sham group (week two: $1.71 \pm 0.60$, week four: $2.34 \pm 1.56$ ) (Figure 4B). The $\mathrm{G}$ group increased the $\triangle \mathrm{BV} / \mathrm{TV}$ by $142 \%$ and $125 \%$ in week two and week four, respectively, while the GO group increased it by $78 \%$ and $137 \%$ in week two and week four, respectively. The G group also significantly increased the value of $\Delta \mathrm{Tb}$.Th (Figure $4 \mathrm{~B}$ ) by $181 \%$ in week two $(0.031 \pm 0.007)$ and $100 \%$ in week four $(0.034 \pm 0.006)$ compared with the sham group (week two: $0.012 \pm 0.003$, week $4: 0.017 \pm 0.009$ ). The GO group increased it by $91 \%$ in week two $(0.021 \pm 0.004)$ and $71 \%$ in week $4(0.029 \pm 0.004)$ compared with the sham group.

\section{Histological Analysis \\ HE Staining}

The HE staining was used to evaluate the changes of bone tissue type and soft tissue (Figure 5). The results at the sixth week showed that typical osteolysis thickened the fibrous tissue in the presence of PS particle and that the morphology changed after engulfing the particles. The results of the $G$ group were similar to those of the PS group, wherein the graphene and GO particles were located within the cell, and multinuclear giant cells were observed to be distributed around the soft tissue (at the arrow).

\section{Masson Trichrome Staining}

Fibrous tissue and bone formation (new bone above the dotted line) were observed with Masson trichrome staining (Figure 6). The results were similar to those of the HE 
A

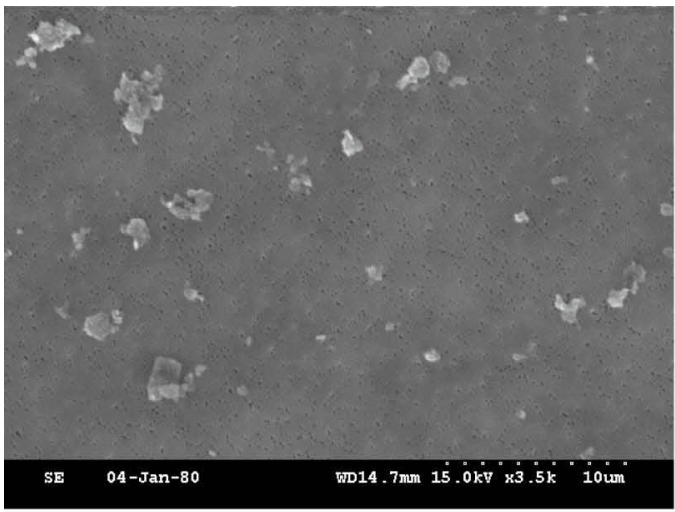

C

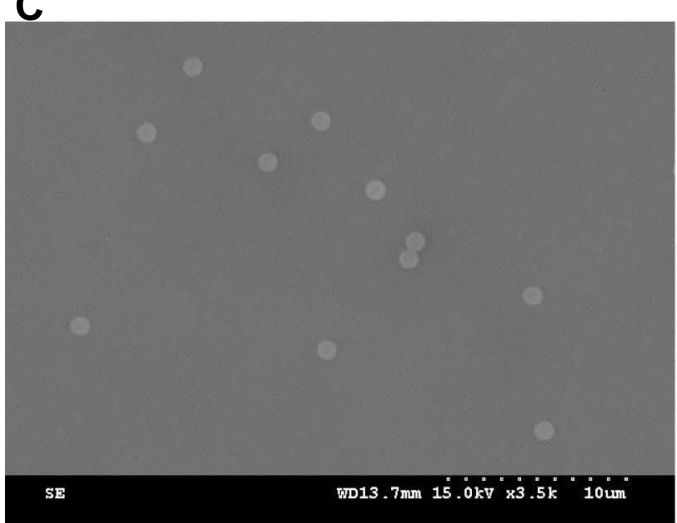

B

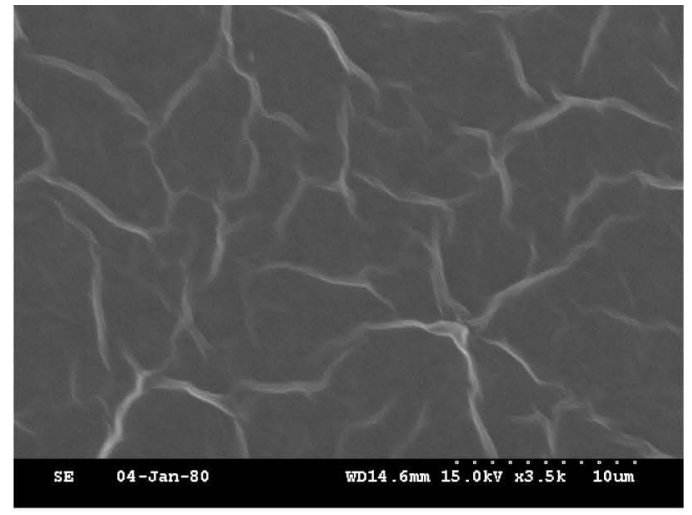

Figure 2 SEM of sub-micrometer particles. (A) Graphene; (B) GO; (C) PS. Note that as the shape of GO is a one-atom-thick sheet, it is hard to determine its mean size and aspect ratio via SEM observation.

A

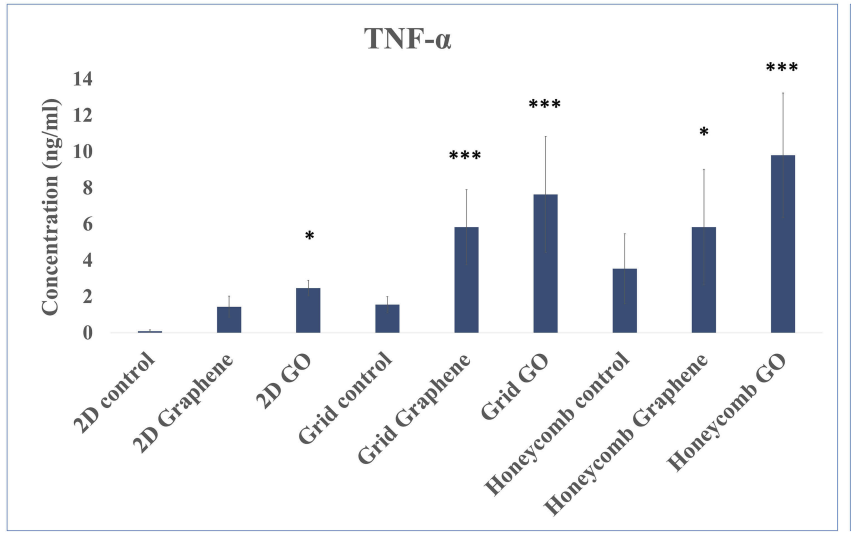

B

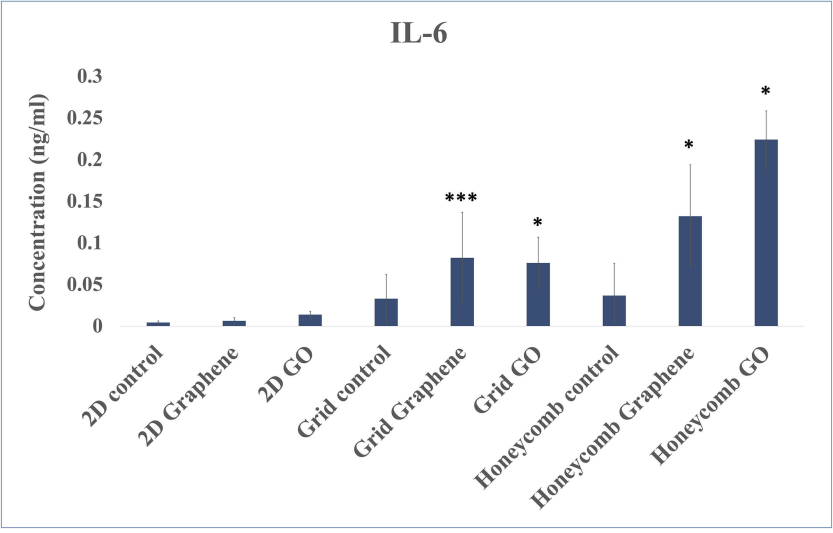

Figure 3 Cytokine secretion of cell treated with graphene or GO in different 2D/3D structures. (A) TNF- $\boldsymbol{\alpha} ;(\mathbf{B})$ IL-6 (mean \pm SD, $* \mathrm{p}<0.05$, $* * * \mathrm{p}<0.00 \mathrm{I})$.

staining. In the bone tissue part, the PS group showed less obvious new bone formation, and the surface of the skull bone was affected by osteolysis and formed cavities. The staining results showed that the $G$ group had obvious new bone formation compared with the GO group. The overall shape of the new bone in the $G$ group was relatively flat, while that of the GO group was more irregular. The GO group had a large cavity and a thinner cavity, and it was observed that the aggregated GO particles were surrounded by newly formed fibrous tissue and bone. 
A
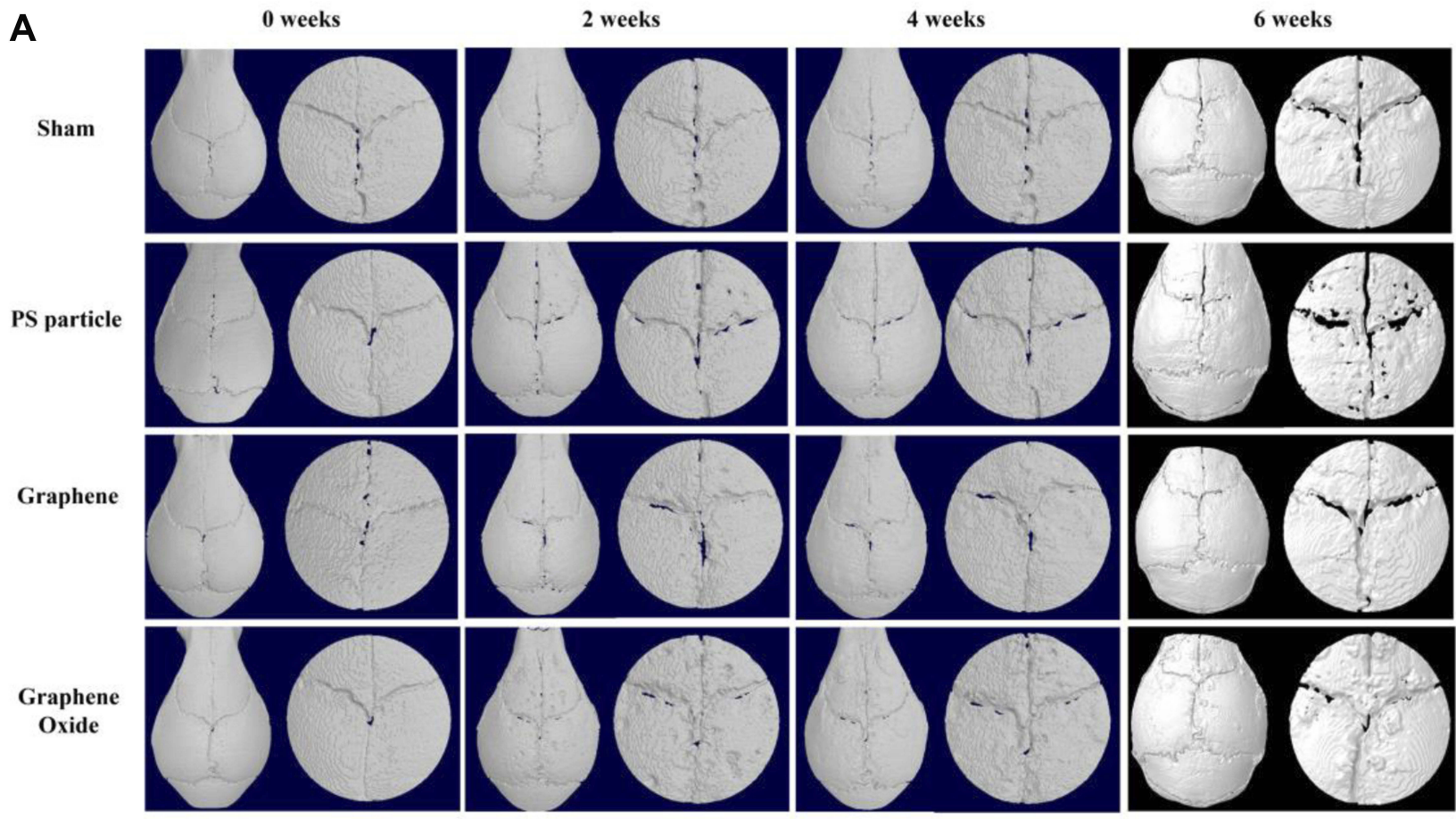

B
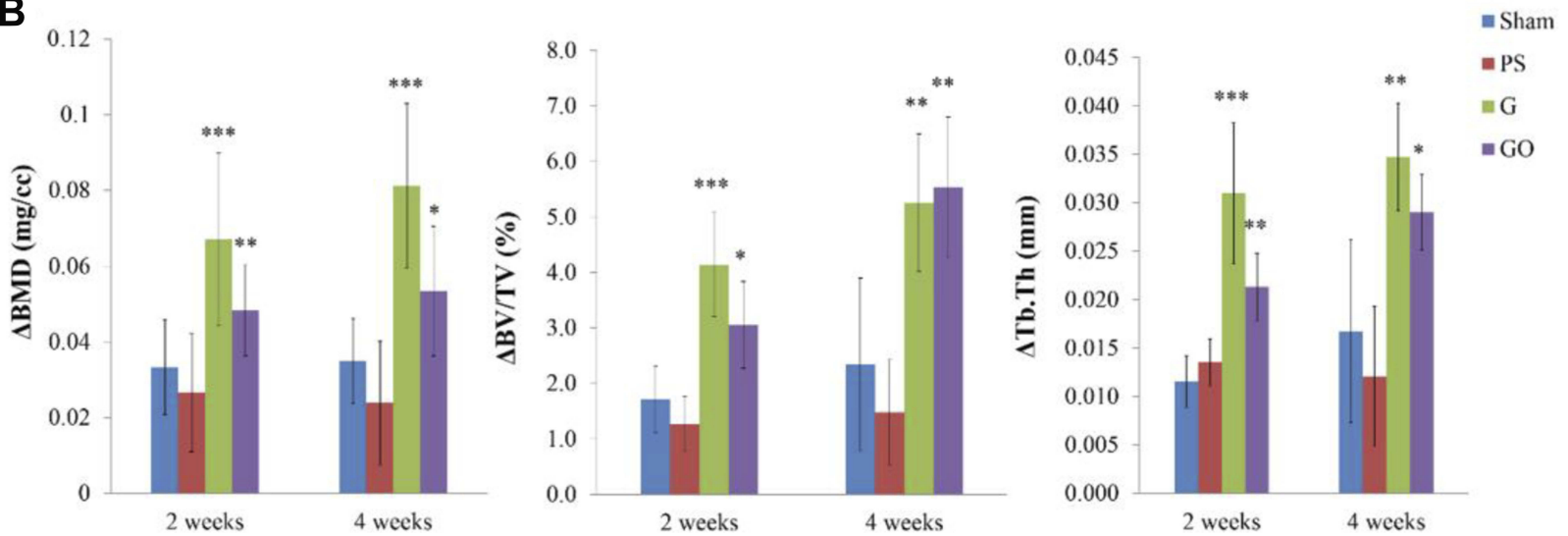

Figure 4 Micro-CT imaging analysis of murine calvarial model treated with different particles. (A) Reconstructed image of whole skull and VOI with the midline suture of the skull. The VOI is defined with a diameter of $5 \mathrm{~mm}$. (B) Bone resorption parameter quantified by micro-CT in calvarial tissues (mean $\pm \mathrm{SD}$, * $\mathrm{P}<0.05$, $* * \mathrm{p}<0.0 \mathrm{I}, * * * \mathrm{p}<0.00 \mathrm{I})$.

\section{TRAP Staining}

The observation and calculation of the osteoclast numbers with TRAP staining showed that the osteoclast numbers of the PS $(45.2 \pm 10.8)$ and $G$ groups $(54.4 \pm 19.1)$ had an increasing trend compared with that of the sham group $(31.3 \pm 13.1)$, but there was no significant difference in any group (Figure 7). The osteoclast number in the GO group (34.4 \pm 21.8$)$ was no different from that of the sham group.

\section{Discussion}

Graphene and its derivatives are novel materials that have been used in various fields in recent years, including the biomedical, ${ }^{16}$ chemical, ${ }^{43}$ and industrial ${ }^{44}$ fields. Due to their excellent mechanical properties, graphene and GO could be incorporated as new materials in artificial joint replacement liners. However, if graphene- and GO-containing implants are used in weight-bearing parts, there could be debris generated by heavy cyclic loading. Whether the debris of graphene and GO affects the human body is an important question that needs to be thoroughly evaluated. Therefore, this study applied well developed in vitro and in vivo osteolysis models to test whether the debris of graphene and GO has any harmful effects in inducing osteolysis.

The results of the in vitro study showed that graphene and GO induced an inflammatory response in the $3 \mathrm{D}$ in vitro 


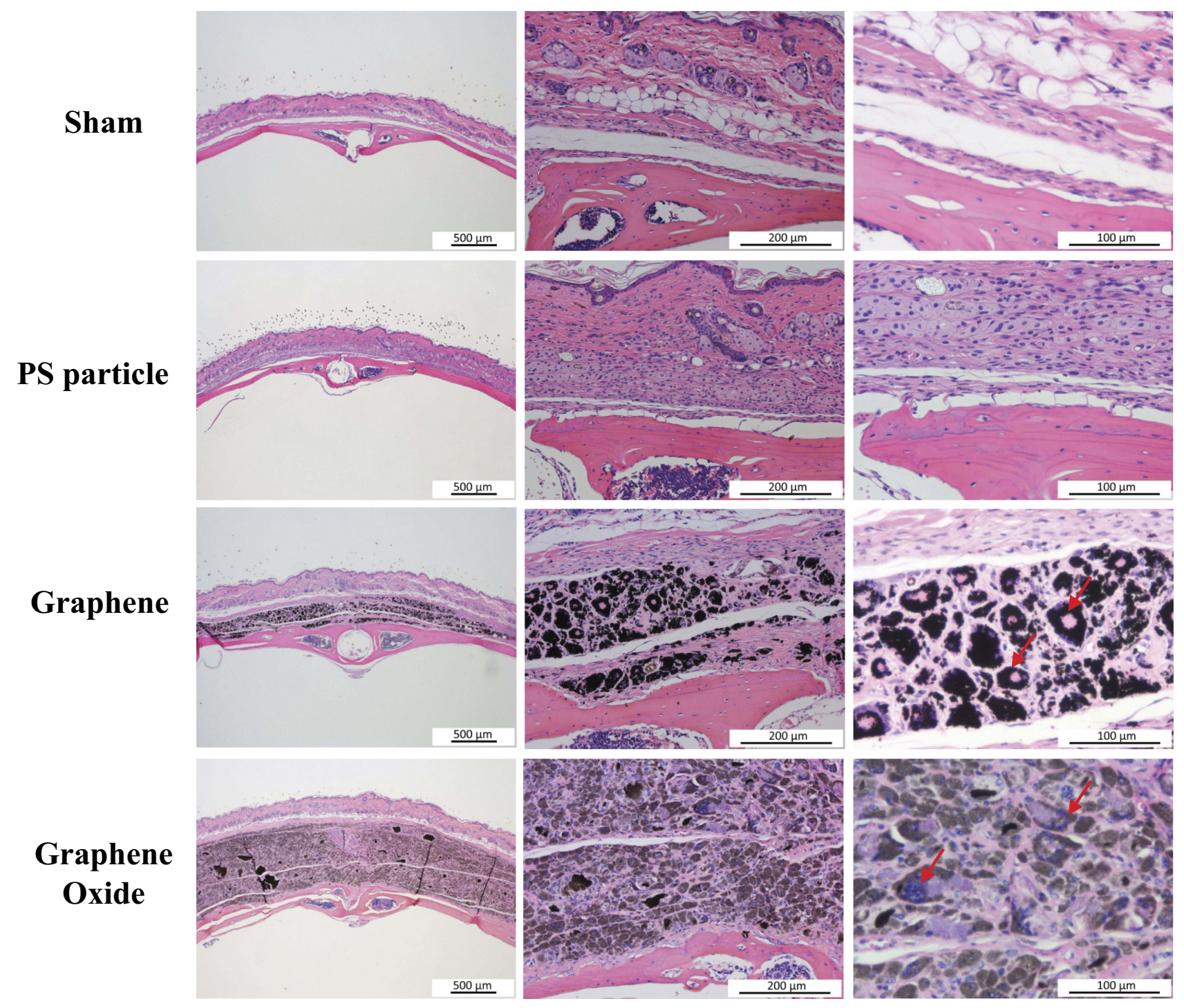

Figure 5 Photomicrograph of hematoxylin and eosin staining in murine calvarial tissues (arrow: multinucleated giant cells).

osteolysis model. These results did not appear to match the in vivo results that indicated that graphene and GO induce osteogenesis rather than osteolysis. However, recent research works have indicated that the inflammatory reaction is essential to achieve bone formation. ${ }^{45,46}$ Research has shown that GO activates macrophage toward the M1 phase through the activation of the OSM signaling pathway, which is beneficial for osteogenesis. ${ }^{47}$ Macrophages are activated to the M1 proinflammatory phase for $72 \mathrm{hrs}$ before turning to the M2 anti-inflammatory phase to promote osteogenesis. ${ }^{48}$ This mechanism might be able to explain why graphene and GO induced inflammation at $72 \mathrm{hrs}$.

In our previous study, we used the same mice calvarial osteolysis model to test the effects of new biomaterial particles of contemporary orthopedic biomaterials
UHMWPE, HXLPE, and VEPE (HXLPE with vitamin E) on the biological responses to the bone interface. ${ }^{32}$ The results indicated that the particles from all the polymers had harmful effects on the calvarial bone, and HXLPE displayed the strongest bone resorption in the micro-CT and histology analysis. ${ }^{32}$ However, the graphene and GO particles appeared to have promising effects on the bone formation, and no osteolytic defects were observed in these calvarial models. The results of the in vivo model showed that the BMD, $\mathrm{Tb}, \mathrm{Th}$, and ratio of BV were increased in the G and GO groups and slightly decreased in the PS group compared with the sham group. In the histological analysis of the Masson Trichrome staining, new bone formation was observed in both the $\mathrm{G}$ and GO groups. Both the micro-CT and histological results 


\section{Sham}

\section{PS particle}

\section{Graphene Oxide}
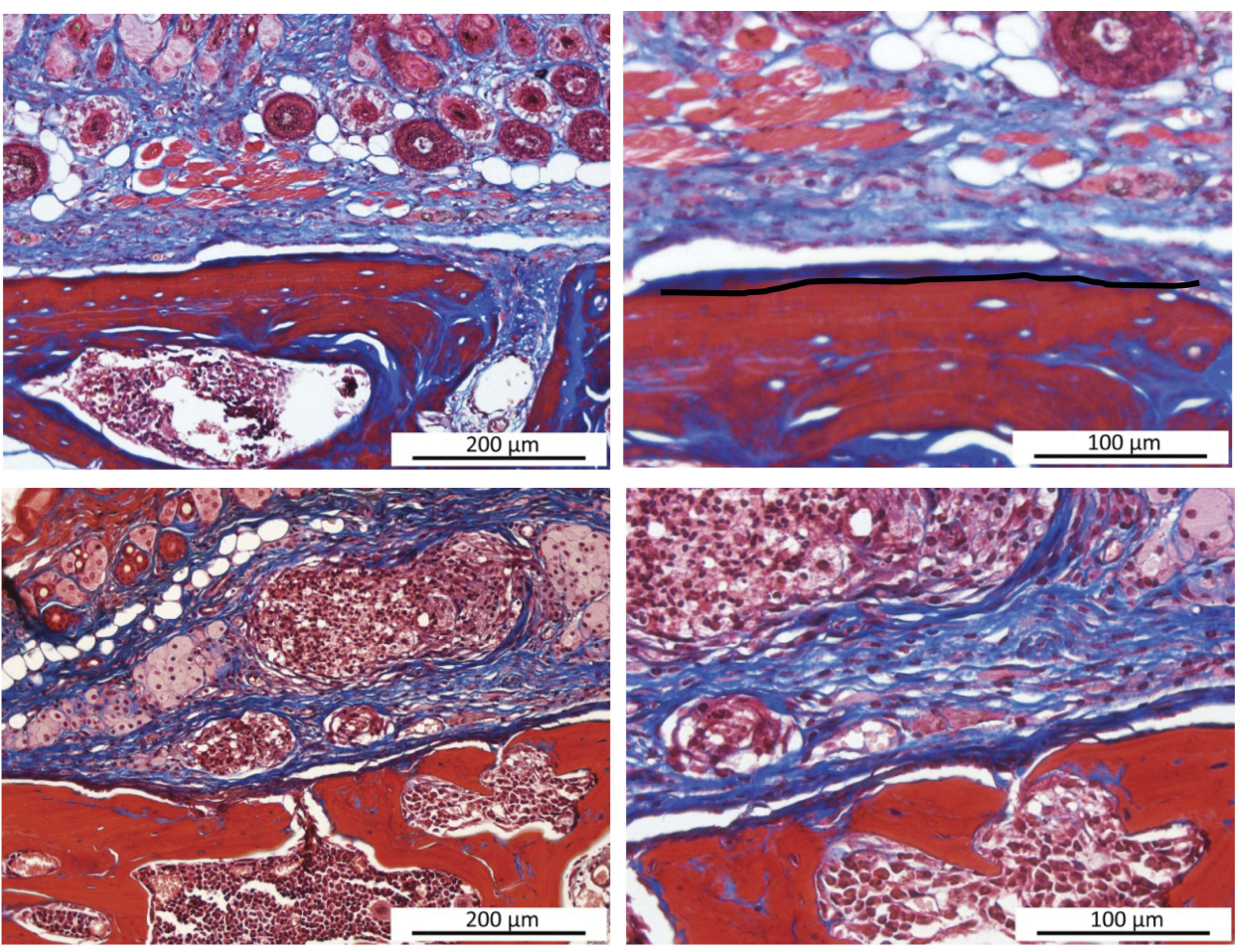

\section{Graphene}
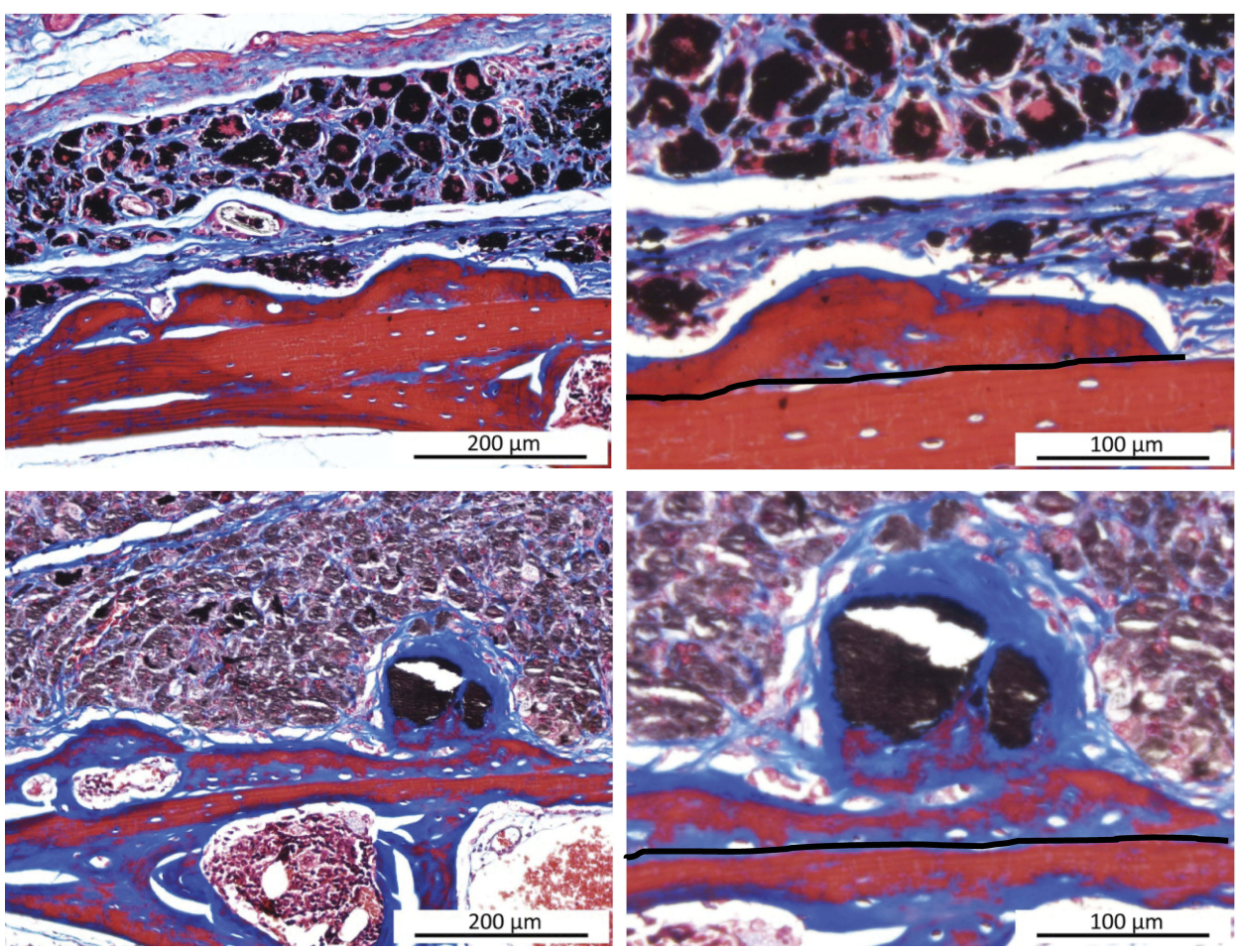

Figure 6 Photomicrograph of Masson trichrome staining in murine calvarial tissues (fibrous tissue: blue; bone: red and blue).

indicated that graphene and GO might have the potential to promote osteogenesis rather than induce osteolysis.

Other research studies regarding the osteogenic effects of graphene and its derivates have shown similar results. Cell proliferation and osteogenic markers were significantly increased in MG63 and MC3T3 cell lines cultured on graphene- and GO-coated Ti disks. ${ }^{23,24}$ The researchers used a chitosan cell scaffold containing $0.25 \%$ GO to fill a $3-\mathrm{mm}$ bone defect in a rat tibia bone defect model for 2 weeks. The results showed 

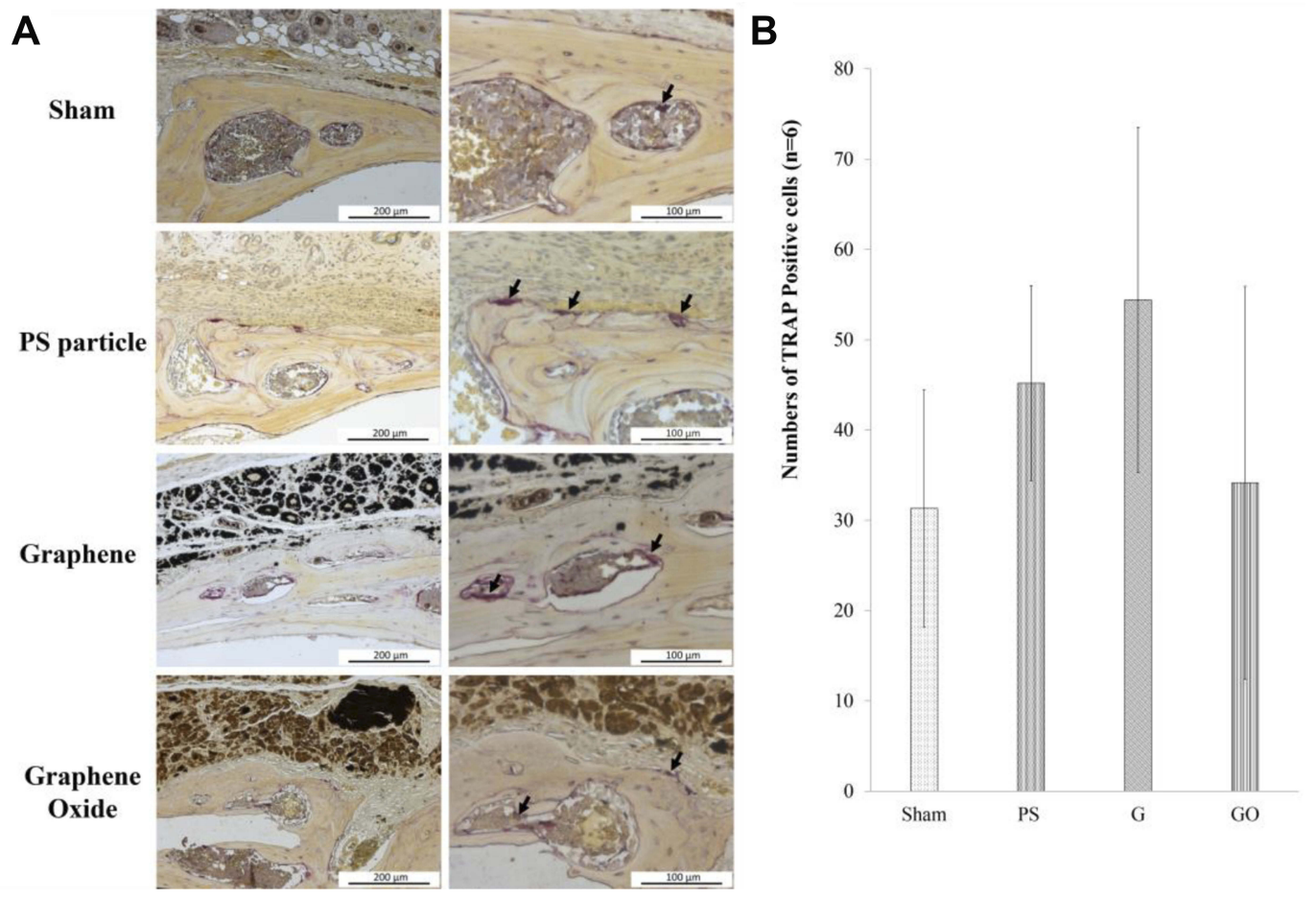

Figure 7 (A) Photomicrograph of TRAP staining in murine calvarial tissues, with osteoclast aggregation indicated by arrows. TRAP immunohistochemistry shows purplestained TRAP + positive osteoclasts. (B) Average number of osteoclasts from each group is presented as the mean \pm SD.

that GO might have osteogenic effects. ${ }^{49}$ In a mouse calvarial bone defect model, a chitosan scaffold with $3 \%$ GO showed significantly higher osteogenic effects than that with a lower concentration $(0.5 \%) .{ }^{50}$ After 18 weeks of implantation of the calvarial bone defect, the group with the chitosan scaffold with 3\% GO showed $60 \%$ new bone formation, significantly higher than that of the $0.5 \%$ GO group (30\%) and control chitosan scaffold group (10\%).

In addition to their osteogenic effects, graphene and its derivates show great promise as biosensors, ${ }^{44,51}$ antibacterial agents, ${ }^{52,53}$ bioimaging tools, ${ }^{54,55}$ and tissue engineer components. ${ }^{56}$ The results from our animal study showed that graphene and GO might be promising candidates for novel orthopedic materials. A possible reason for this might concern their surface properties, such as the surface charge of the particles, which plays an important role in their effects on cell behavior. ${ }^{57,58}$ Their high surface area also helps promote cell adhesion and growth..$^{27,28}$

This study had some limitations. First, it used a particleinduced murine calvarial osteolysis model, though osteolysis often occurs in proximity to prostheses. Thus, the observed effects may not strictly conform to clinical conditions. However, such models are widely used in osteolysis evaluations and are useful for assessing the effects of different materials on the osteolytic response. ${ }^{59}$ It was hard to obtain quantitative results from the histological analysis of the animal model HE and TRAP staining. Thus, the current study used micro-CT to quantify the differences of bone mass among the groups. Second, in a real-life scenario, the wear debris should contain both polymer and graphene/GO. However, the current study used pure graphene and GO particles to determine the biological response, as the optimized percentage of graphene- and GO-containing polymers for medical use in the future is unknown. According to previous studies, polymers used in the orthopedic field, including polyethylene and PS debris, have been shown to induce osteolysis. ${ }^{32,35}$ The current study used $1 \mathrm{mg}$ of pristine graphene and GO particles, ie, the same amount we used in our previous study. ${ }^{32,34}$ We found that these submicron-sized particles did not induce any osteolytic pores on the calvarias. Further tribology analyses of polymer-containing graphene or GO would be helpful to obtain the real-size distribution. Finally, the in vitro tests were only $72 \mathrm{hrs}$ long; if the tests had been extended for a longer period, osteogenic effects might have been observed. Thus, the 3D culture system might need to be adjusted for a longer culture time in the future. Aside from the above limitations, the current results demonstrate 
that graphene and GO particles do not have adverse effects on bone.

\section{Conclusion}

In the murine calvarial osteolysis model studied here, the submicron-sized particles of graphene and GO had no adverse effect on the bone. In addition to being incorporated into orthopedic liners, they can be expected to be applied in other fields due to their potential benefit of osteogenesis. However, further biological and biomechanical characteristics of the particles should be determined to ensure their safety and effectiveness before marketing.

\section{Acknowledgment}

The authors are pleased to acknowledge the financial support from Ministry of Science of Technology in Taiwan (MOST 107-2314-B-002). We also thank the Taiwan Mouse Clinic which is funded by National Research Program for Biopharmaceuticals (NRPB) at Ministry of Science and Technology (MOST) of Taiwan and Tron Medicalbio Corp. for technical support with the micro-CT experiments and the experimental support by the Electron Microscope Laboratory of MacKay Memorial Hospital.

\section{Author Contributions}

All authors made substantial contributions to conception and design, acquisition of data, or analysis and interpretation of data; took part in drafting the article or revising it critically for important intellectual content; gave final approval of the version to be published; and agree to be accountable for all aspects of the work.

\section{Disclosure}

The authors report no conflicts of interest in this work.

\section{References}

1. Ma HM, Lu YC, Ho FY, Huang CH. Long-term results of total condylar knee arthroplasty. $J$ Arthroplasty. 2005;20(5):580-584. doi:10.1016/j.arth.2005.04.006

2. Milligan DJ, O'Brien S, Doran E, Gallagher NE, Beverland DE. Twentyyear survivorship of a cemented mobile bearing total knee arthroplasty Knee. 2019;26(4):933-940. doi:10.1016/j.knee.2019.06.004

3. Amstutz HC, Campbell P, Kossovsky N, Clarke IC. Mechanism and clinical significance of wear debris-induced osteolysis. Clin Orthop Relat Res. 1992;276:7-18.

4. DeHeer DH, Engels JA, DeVries AS, Knapp RH, Beebe JD. In situ complement activation by polyethylene wear debris. J Biomed Mater Res. 2001;54(1):12-19. doi:10.1002/(ISSN)1097-4636

5. McKellop H, Shen FW, DiMaio W, Lancaster JG. Wear of gamma-crosslinked polyethylene acetabular cups against roughened femoral balls. Clin Orthop Relat Res. 1999;369(369):73-82. doi:10.1097/00003086-199912000-00008
6. Capello WN, D'Antonio JA, Ramakrishnan R, Naughton M. Continued improved wear with an annealed highly cross-linked polyethylene. Clin Orthop Relat Res. 2011;469(3):825-830. doi:10. 1007/s11999-010-1556-5

7. Dion NT, Bragdon C, Muratoglu O, Freiberg AA. Durability of highly cross-linked polyethylene in total hip and total knee arthroplasty. Orthop Clin North Am. 2015;46(3):321-327, ix. doi: 10.1016 /j.ocl.2015.02.001

8. Kurtz SM, Gawel HA, Patel JD. History and systematic review of wear and osteolysis outcomes for first-generation highly crosslinked polyethylene. Clin Orthop Relat Res. 2011;469(8):2262-2277. doi:10.1007/s11999-011-1872-4

9. Lambert B, Neut D, van der Veen HC, Bulstra SK. Effects of vitamin E incorporation in polyethylene on oxidative degradation, wear rates, immune response, and infections in total joint arthroplasty: a review of the current literature. Int Orthop. 2019;43(7):1549-1557. doi:10.1007/s00264-018-4237-8

10. Chen Y, Hallab NJ, Liao YS, Narayan V, Schwarz EM, Xie C. Antioxidant impregnated ultra-high molecular weight polyethylene wear debris particles display increased bone remodeling and a superior osteogenic: osteolyticprofile vs. conventional UHMWPE particles in a murine calvaria model. J Orthop Res. 2016;34 (5):845-851. doi:10.1002/jor.23080

11. Ponzio DY, Weitzler L, deMeireles A, Esposito CI, Wright TM, Padgett DE. Antioxidant-stabilized highly crosslinked polyethylene in total knee arthroplasty: a retrieval analysis. Bone Joint J. 2018;100-B(10):1330-1335. doi:10.1302/0301-620X.100B10.BJJ2018-0061.R2

12. Barrack RL. Retrieval analysis of an early fracture of a vitamin E-stabilized tibial liner in total knee arthroplasty: a case report. JBJS Case Connect. 2013;3(2 Suppl 2):1-6. doi:10.2106/JBJS.CC. L.00276

13. Tadyszak K, Wychowaniec JK, Litowczenko J. Biomedical applications of graphene-based structures. Nanomaterials (Basel, Switzerland). 2018;8(11):944. doi:10.3390/nano8110944

14. Han U, Seo Y, Hong J. Effect of $\mathrm{pH}$ on the structure and drug release profiles of layer-by-layer assembled films containing polyelectrolyte, micelles, and graphene oxide. Sci Rep. 2016;6:24158. doi:10.1038/ srep24158

15. Singh DP, Herrera CE, Singh B, Singh S, Singh RK, Kumar R. Graphene oxide: an efficient material and recent approach for biotechnological and biomedical applications. Mater Sci Eng C Mater Biol Appl. 2018;86:173-197. doi:10.1016/j.msec.2018.01.004

16. Banerjee AN. Graphene and its derivatives as biomedical materials: future prospects and challenges. Interface Focus. 2018;8 (3):20170056. doi:10.1098/rsfs.2017.0056

17. Luo Z, Hu X, Tian X, et al. Structure-property relationships in graphene-based strain and pressure sensors for potential artificial intelligence applications. Sensors (Basel). 2019;19(5):1250. doi:10. $3390 / \mathrm{s} 19051250$

18. Zhao K, Zhang T, Chang H, et al. Super-elasticity of three-dimensionally cross-linked graphene materials all the way to deep cryogenic temperatures. Sci Adv. 2019;5(4):eaav2589. doi:10.1126/sciadv.aav2589

19. Zhu Y, Murali S, Cai W, et al. Graphene and graphene oxide: synthesis, properties, and applications. Adv Mater. 2010;22 (35):3906-3924. doi:10.1002/adma.201001068

20. Gao W. The chemistry of graphene oxide. In: Gao W, editor. Graphene Oxide: Reduction Recipes, Spectroscopy, and Applications. Cham: Springer International Publishing; 2015:61-95.

21. Chiu NF, Huang TY. Sensitivity and kinetic analysis of graphene oxide-based surface plasmon resonance biosensors. Sens Actuators B Chem. 2014;197:35-42. doi:10.1016/j.snb.2014.02.033

22. Stankovich S, Dikin DA, Piner RD, et al. Synthesis of graphene-based nanosheets via chemical reduction of exfoliated graphite oxide. Carbon. 2007;45(7):1558-1565. doi:10.1016/j.carbon.2007.02.034 
23. Dubey N, Ellepola K, Decroix FED, et al. Graphene onto medical grade titanium: an atom-thick multimodal coating that promotes osteoblast maturation and inhibits biofilm formation from distinct species. Nanotoxicology. 2018;12(4):274-289. doi:10.1080/17435390.2018.143 4911

24. Ren L, Pan S, Li H, et al. Effects of aspirin-loaded graphene oxide coating of a titanium surface on proliferation and osteogenic differentiation of MC3T3-E1 cells. Sci Rep. 2018;8(1):15143. doi:10.1038/ s41598-018-33353-7

25. Pang W, Ni Z, Chen G, Huang G, Huang H, Zhao Y. Mechanical and thermal properties of graphene oxide/ultrahigh molecular weight polyethylene nanocomposites. RSC Adv. 2015;5:63063-63072. doi:10.1039/C5RA11826C

26. Wang W, Caetano G, Chiang W-H, et al. Morphological, mechanical and biological assessment of PCL/pristine graphene scaffolds for bone regeneration. Int J Bioprinting. 2016;2:95-105. doi:10.18063/ IJB.2016.02.009

27. Frahs SM, Reeck JC, Yocham KM, et al. Prechondrogenic ATDC5 cell attachment and differentiation on graphene foam; modulation by surface functionalization with fibronectin. ACS Appl Mater Interfaces. 2019;11(45):41906-41924. doi:10.1021/acsami.9b14670

28. Amani H, Mostafavi E, Arzaghi H, et al. Three-dimensional graphene foams: synthesis, properties, biocompatibility, biodegradability, and applications in tissue engineering. ACS Biomater Sci Eng. 2019;5 (1):193-214. doi:10.1021/acsbiomaterials.8b00658

29. Pang L, Dai C, Bi L, Guo Z, Fan J. Biosafety and antibacterial ability of graphene and graphene oxide in vitro and in vivo. Nanoscale Res Lett. 2017;12(1):564. doi:10.1186/s11671-017-2317-0

30. Jaworski S, Strojny B, Sawosz E, et al. Degradation of mitochondria and oxidative stress as the main mechanism of toxicity of pristine graphene on U87 glioblastoma cells and tumors and HS-5 cells. Int J Mol Sci. 2019;20(3):650. doi:10.3390/ijms20030650

31. Franqui LS, De Farias MA, Portugal RV, et al. Interaction of graphene oxide with cell culture medium: evaluating the fetal bovine serum protein corona formation towards in vitro nanotoxicity assessment and nanobiointeractions. Mater Sci Eng C Mater Biol Appl. 2019;100:363-377. doi:10.1016/j.msec.2019.02.066

32. Huang $\mathrm{CH}$, Lu YC, Chang TK, et al. In vivo biological response to highly cross-linked and vitamin e-doped polyethylene-a particleinduced osteolysis animal study. J Biomed Mater Res B Appl Biomater. 2016;104(3):561-567. doi:10.1002/jbm.b.33426

33. Lu YC, Yeh ST, Huang CH, et al. Applications for additive manufacturing technology in cell culture: particleinduced osteolysis in vitro system. Orthopaedic Research Society 2018 Annual Meeting; March 10-13, 2018; New Orleans, LA.

34. Lu YC, Chang TK, Yeh ST, et al. The potential role of strontium ranelate in treating particle-induced osteolysis. Acta Biomater. 2015;20:147-154. doi:10.1016/j.actbio.2015.03.034

35. Ortiz SG, Ma T, Regula D, Smith RL, Goodman SB. Continuous intramedullary polymer particle infusion using a murine femoral explant model. J Biomed Mater Res B Appl Biomater. 2008;87 (2):440-446. doi:10.1002/jbm.b.31122

36. Hahn DW, Wolfarth DL, Parks NL. Characterization of submicron polyethylene wear debris from synovial-fluid samples of revised knee replacements using a light-scattering technique. $J$ Biomed Mater Res. 1996;31:355-63. doi:10.1002/(SICI)1097-4636(199607)31:3<355:: AID-JBM9>3.0.CO;2-LI

37. Endotoxin Testing Recommendations for Single-Use Intraocular Ophthalmic Devices. Draft Guidance for Industry and Food and Drug Administration Staff; 2014. Available from: https://www.fda. gov/regulatory-information/search-fda-guidance-documents/endo toxin-testing-recommendations-single-use-intraocular-ophthalmicdevices.
38. Gao X, Ge J, Li W, Zhou W, Xu L. LncRNA KCNQ1OT1 ameliorates particle-induced osteolysis through inducing macrophage polarization by inhibiting miR-21a-5p. Biol Chem. 2018;399(4):375-386. doi:10.1515/hsz-2017-0215

39. Jiao Z, Xu W, Zheng J, et al. Kaempferide prevents titanium particle induced osteolysis by suppressing JNK activation during osteoclast formation. Sci Rep. 2017;7(1):16665. doi:10.1038/s41598-01716853-w

40. Wei HJ, Chen $\mathrm{CH}$, Lee WY, et al. Bioengineered cardiac patch constructed from multilayered mesenchymal stem cells for myocardial repair. Biomaterials. 2008;29(26):3547-3556. doi:10.1016/j. biomaterials.2008.05.009

41. Chen $\mathrm{CH}$, Tsai CC, Chen W, et al. Novel living cell sheet harvest system composed of thermoreversible methylcellulose hydrogels. Biomacromolecules. 2006;7(3):736-743. doi:10.1021/bm0506400

42. Wang $\mathrm{CC}$, Chen $\mathrm{CH}$, Lin WW, et al. Direct intramyocardial injection of mesenchymal stem cell sheet fragments improves cardiac functions after infarction. Cardiovasc Res. 2008;77(3):515-24. doi:10.1093/cvr/ cvm046

43. Nouri N, Khorram P, Sereshti H. Applications of three-dimensional graphenes for preconcentration, extraction, and sorption of chemical species: a review. Mikrochim Acta. 2019;186(4):232. doi:10.1007/ s00604-019-3324-x

44. Wang Z, Gao H, Zhang Q, Liu Y, Chen J, Guo Z. Recent advances in $3 \mathrm{D}$ graphene architectures and their composites for energy storage applications. Small. 2019;15(3):e1803858.

45. Mu Y, Yang L, Li C, et al. Role of inflammatory factors in regulation of osteogenesis in tissue-engineered bone. Osteogenesis and Bone Regeneration. 2019. doi:10.5772/intechopen.81153

46. van Well GTJ, Daalderop LA, Wolfs T, Kramer BW. Human perinatal immunity in physiological conditions and during infection. Mol Cell Pediatr. 2017;4(1):4. doi:10.1186/s40348-017-0070-1

47. Xue D, Chen E, Zhong $\mathrm{H}$, et al. Immunomodulatory properties of graphene oxide for osteogenesis and angiogenesis. Int J Nanomedicine. 2018;13:5799-5810. doi:10.2147/IJN

48. Loi F, Cordova LA, Zhang R, et al. The effects of immunomodulation by macrophage subsets on osteogenesis in vitro. Stem Cell Res Ther. 2016;7:15. doi:10.1186/s13287-016-0276-5

49. Saravanan S, Chawla A, Vairamani M, Sastry TP, Subramanian KS, Selvamurugan N. Scaffolds containing chitosan, gelatin and graphene oxide for bone tissue regeneration in vitro and in vivo. Int $\mathrm{J} \mathrm{Biol}$ Macromol. 2017;104(Pt $\quad$ B):1975-1985. doi:10.1016/j.ijbiomac. 2017.01.034

50. Hermenean A, Codreanu A, Herman H, et al. Chitosan-graphene oxide 3D scaffolds as promising tools for bone regeneration in critical-size mouse calvarial defects. Sci Rep. 2017;7(1):16641. doi:10.1038/s41598-017-16599-5

51. Bo X, Zhou M, Guo L. Electrochemical sensors and biosensors based on less aggregated graphene. Biosens Bioelectron. 2017;89(Pt 1):167-186. doi:10.1016/j.bios.2016.05.002

52. Karahan HE, Wiraja C, Xu C, et al. Graphene materials in antimicrobial nanomedicine: current status and future perspectives. Adv Healthc Mater. 2018;7(13):e1701406. doi:10.1002/adhm.v7.13

53. Xia MY, Xie Y, Yu CH, et al. Graphene-based nanomaterials: the promising active agents for antibiotics-independent antibacterial applications. J Control Release. 2019;307:16-31. doi:10.1016/j. jconrel.2019.06.011

54. Gu H, Tang H, Xiong P, Zhou Z. Biomarkers-based biosensing and bioimaging with graphene for cancer diagnosis. Nanomaterials (Basel). 2019;9(1):130. doi:10.3390/nano9010130

55. Luo Y, Li Z, Zhu C, et al. Graphene-like metal-free 2D nanosheets for cancer imaging and theranostics. Trends Biotechnol. 2018;36 (11):1145-1156. doi:10.1016/j.tibtech.2018.05.012 
56. Eivazzadeh-Keihan R, Maleki A, de la Guardia M, et al. Carbon based nanomaterials for tissue engineering of bone: building new bone on small black scaffolds: a review. $J$ Adv Res. 2019;18:185-201. doi:10.1016/j.jare.2019.03.011

57. Amani H, Arzaghi H, Bayandori M, et al. Controlling cell behavior through the design of biomaterial surfaces: a focus on surface modification techniques. Adv Mater Interfaces. 2019;6(13):1900572. doi:10.1002/admi.v6.13
58. Amani H, Mostafavi E, Alebouyeh MR, et al. Would Colloidal gold nanocarriers present an effective diagnosis or treatment for ischemic stroke? Int J Nanomedicine. 2019;14:8013-8031. doi:10.2147/IJN. S210035

59. Nich C, Rao AJ, Valladares RD, et al. Role of direct estrogen receptor signaling in wear particle-induced osteolysis. Biomaterials. 2013;34 (3):641-650. doi:10.1016/j.biomaterials.2012.10.030
International Journal of Nanomedicine

\section{Publish your work in this journal}

The International Journal of Nanomedicine is an international, peerreviewed journal focusing on the application of nanotechnology in diagnostics, therapeutics, and drug delivery systems throughout the biomedical field. This journal is indexed on PubMed Central, MedLine, CAS, SciSearch ${ }^{\mathbb{R}}$, Current Contents ${ }^{\mathbb{R}} /$ Clinical Medicine,
Journal Citation Reports/Science Edition, EMBase, Scopus and the Elsevier Bibliographic databases. The manuscript management system is completely online and includes a very quick and fair peer-review system, which is all easy to use. Visit http://www.dovepress.com/ testimonials.php to read real quotes from published authors. 